counter. The relative rate is given by

$$
C\left(\mu_{0} x\right)=\left[\int_{0}^{E^{\prime} \max } f^{\prime}\left(E^{\prime}, \mu_{0} x\right) \epsilon\left(E^{\prime}\right) d E^{\prime}+\epsilon\left(E_{0}\right) e^{-\mu_{0} x}\right] K,
$$

where $\epsilon\left(E^{\prime}\right)$ is the efficiency ${ }^{8}$ of the Geiger counter and $K$ is a normalization constant.

Geiger counter observations of the relative counting rate produced by a $\mathrm{Cs}^{137}$ source in water are represented by the solid curve of Fig. 7 . Statistics of the data were of the order of 1 percent and are not indicated on the curves. The circles represent the relative counting rate $C\left(\mu_{0} x\right)$ as computed from Eq. (4).

\section{ACKNOWLEDGMENTS}

The authors wish to thank Dr. E. H. Krause for his interest and support and express their appreciation

${ }^{8}$ Bradt et al., Helv. Phys. Acta 19, 77 (1946).

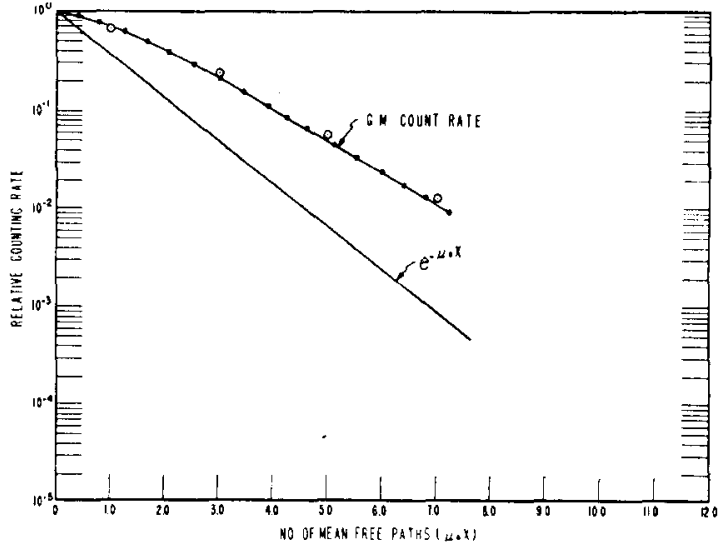

FIG. 7. Comparison of experimental Geiger-Mueller counting data and theoretical counting data at various penetration depths.

to Mr. T. H. DeRieux for aid in performing the experiments.

\title{
Bistatic Radar Cross Sections of Surfaces of Revolution*
}

K. M. Siegel, H. A. Alperin, R. R. Bonkowski, J. W. Crispin, A. L. Maffett, C. E. Schensted, and I. V. Schensted Willow Run Research Center, University of Michigan, Ann Arbor, Michigan

\author{
(Received April 9, 1954)
}

\begin{abstract}
The results obtained by applying the current-distribution method to the problem of approximating the scattering cross section, when the transmitter and receiver are separated, are presented for several simple geometric configurations. The method is applied for the case in which the transmitter is located on the axis of revolution and the ratio $l / \lambda$ is large, where $l$ is a "characteristic dimension" of the body and $\lambda$ is the wavelength. These results indicate that in most of the cases considered the cross section increases as the angle between the receiver and the transmitter increases.
\end{abstract}

\section{INTRODUCTION}

$I^{\mathrm{N}}$ $\mathrm{N}$ this paper, bistatic radar cross sections of simple configurations are obtained by applying an approximation method. In most of the cases considered the configuration is a surface of revolution, the transmitter is located on the axis of symmetry of the body, the polarization is specified, and the position of the receiver is allowed to vary in a plane containing the axis of the body.

The physical description of bistatic cross sections differs from that of monostatic cross sections in that the receiver and the transmitter are permitted to be

\footnotetext{
* This paper is a condensation of the report UMM-115, "Studies in radar cross-sections VIII-theoretical cross-sections as a function of separation angle between transmitter and receiver at small wavelengths" by Siegel, Alperin, Bonkowski, Crispin, Maffett, Schensted, and Schensted, Willow Run Research Center University of Michigan, October, 1953. In addition, portions of this paper were presented in a talk "Determination of scattering cross-sections for the case of separated transmitter and receiver" by Siegel, Bonkowski, Crispin, and I. V. Schensted, presented at the Symposium on Microwave Optics, McGill University, Montreal, Canada, 1953.
}

located at separate positions. To specify the bistatic radar cross section of a body for a general location of transmitter and receiver more than one angle is required. However for most cases discussed in this paper, one angle, the angle $\beta$ shown in Fig. 1, suffices. The bistatic radar cross section, therefore, is denoted by $\sigma(\beta)$. It is evident that the monostatic backscattering cross section is a special case $(\beta=0)$ of the bistatic cross section and can be denoted by $\sigma(0)$.

The approximation technique used is the currentdistribution or physical-optics method. This method is applicable when the wavelength of the incident radiation is small with respect to the characteristic dimension

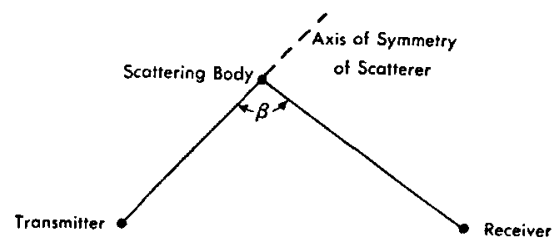

FIG. 1. The angle $\beta$. 
of the body. In using any approximation method it is desirable to know how the results obtained from it compare with the physically expected or experimental results. In determining the monostatic radar cross section, when the wavelength is much less than the characteristic dimension of the body, the currentdistribution method has provided many results which are in close agreement with experiment and with exact theoretical solutions.

The current-distribution method has been subjected to much criticism on both theoretical and experimental grounds. ${ }^{1,2}$ For example, reasoning based on the assumptions of the current-distribution method would lead to the conclusion that this method should not be used when the surface has a point discontinuity. However, the method predicts the nose-on backscattering cross section of a semi-infinite cone to within experimental accuracies, if not exactly.,3

When solving far-zone problems for which the bodies are infinite in the direction of propagation, Abelian limit processes usually are convenient unless the configuration's tangent at infinity is parallel or perpendicular to the propagation vector. The limit process is commonly used, because it is not possible to get infinitely far from an infinite body. If the viscosity or conductivity of the medium is introduced into the problem (although these quantities may be negligible), then the problem becomes formally the same as the previous one with the objection to the limit process removed. In this paper, an Abelian limit process is used in the discussion of the semi-infinite cone.

Some doubt has been thrown on the applicability of the physical-optics approximations to electromagnetic scattering problems in the past by the assertion that these approximations would always be in error by more than the corresponding physical-optics approximations to the acoustic problem. At least one apparent contradiction to this assertion is presented in references 3 and 4 , where it is shown that physicaloptics predicts the exact electromagnetic cross section for cones of small angle, whereas the same approximate methods predict a value four times as great as that predicted by the acoustic wave equation.

Although the quantitative theoretical explanation of this factor of four cannot be elaborated at the present time, it is clear that such a disagreement should not be unexpected. The acoustic wave equation which

\footnotetext{
${ }^{1}$ B. B. Baker and E. I. Copson, The Mathematical Theory of Huygen's Principle (Oxford University Press, New York, 1950), second edition.

${ }^{2}$ Symposium on Microwave Optics (Eaton Electronics Research Laboratory, McGill University, Montreal, Canada, 1953), Vols. I and II.

${ }^{3}$ Siegel, Alpern, Crispin, Hunter, Kleinman, Orthwein, and Schensted, "Studies in radar cross-sections IV-comparison between theory and experiment of the cross-section of a cone," Willow Run Research Center, University of Michigan, Report No. UMM-92 (February, 1953).

4 Siegel, Crispin, and Schensted, J. Appl. Phys. 26, 309 (1955).
}

has been used is merely an approximation to the exact equation of the motion. This approximation is valid only when the wavelength is large (in comparison to the dimensions of the scatterer), because the influence of viscosity in acoustics is greatest at small wavelengths. ${ }^{5}$ However, the physical-optics approximation is valid only when the wavelength is small in comparison to the dimensions of the scatterer. Hence, the physicaloptics approximation cannot be expected to reproduce accurately the real situation of acoustic scattering at any wavelength, whereas the same approximation can be expected to reproduce accurately the real situation in electromagnetic scattering whenever the wavelength is small.

Although in the above only an infinite body was under discussion, there is nothing in the argument which requires that the surface be infinite; the same type of conclusion would be reached for a finite body in a real fluid.

The cone result shows that it is possible to treat by the current-distribution method at least some bodies which have point discontinuities. There is no proof available at the present time that pointed bodies in general can be treated by physical-optics. Such a proof would require a stronger basis than the present existence and uniqueness proofs in electromagnetic theory, since the papers of Weyl and Müller ${ }^{6,7}$ and the Fredholm theory are based on the assumption that the body is "sufficiently smooth."

In addition to the cone solution, a point (or rather four such singularities) has been treated by Kouyoumjian in his solution for the cross section of a square flat plate $^{8}$ by the Levine-Schwinger variational procedure. ${ }^{9}$ The physical-optics result was in fairly good agreement with the variational result in the region where the wavelength is equal to, or less than, the length of the side of the plate. Kouyoumjian's experimental results agree more closely with the variational curve than with the physical-optics curve, but no great error would be made if the optics result was used.

In addition, the physical-optics determination of the nose-on backscattering cross section of an ogive (still another pointed body) is also in close agreement with experimental results (see reference 3 ).

\section{THE METHOD FOR DETERMINING BISTATIC CROSS SECTIONS}

If the surface of the scattering body is assumed to be perfectly conducting, the equation for the scattered

${ }^{5} \mathrm{~J}$. W. S. Rayleigh, The Theory of Sound (Dover Publications, New York, 1945), second edition.

${ }^{8}$ H. Weyl, Math. Z. 55, 187-198 (1952)

${ }^{7}$ C. Müller, Math. Z. 56, 80-83 (1952).

8 R. G. Kouyoumjian, "Calculation of the echo area of several scatterers of simple geometry by the variational method," Symposium on Microwave Optics (Eaton Electronics Research Laboratory, McGill University, Montreal, Canada, 1953), Vol. I.

${ }^{9} \mathrm{H}$. Levine and J. Schwinger, Commun. Pure Appl. Math. Vol. III, No. 4, p. 355 (December, 1950). 
magnetic field ${ }^{10}$ can be written as

$$
\mathbf{H}_{s c}=\frac{1}{4 \pi} \int_{\mathrm{S}}\left(\mathbf{n} \times \mathbf{H}_{t}\right) \times \nabla\left(\frac{e^{-i k R}}{R}\right) d S,
$$

where $\mathbf{H}_{s c}=$ the scattered magnetic field vector, $\mathbf{n}=$ the unit normal to the surface, $\mathbf{H}_{t}=$ the tangential component of the magnetic field on the scattering surface, $R=$ the distance separating the receiver and the integration point, $k=2 \pi / \lambda(\lambda=$ wavelength), and $S=$ the region of integration $=$ the entire surface of the scatterer.

Under physical-optics assumptions, $\mathbf{H}_{t}$ can be approximated as twice the tangential component of the incident magnetic field on the "illuminated" side of the body and zero on the "shadow" side of the body. $\dagger$ Letting the incident magnetic field have a magnitude $H_{0}$ and a direction a, then according to this approximation

$\mathbf{H}_{t}=2 \mathbf{i}_{t} H_{0} e^{-i k(\mathrm{k} \cdot \mathrm{r})}$

$\mathbf{H}_{t}=0$

on the illuminated side of the body,

on the shadow side of the body,

where $\mathbf{k}=\mathrm{a}$ unit vector directed from the transmitter, assumed to be infinitely far away, to the origin of the coordinate system; $\mathbf{r}=$ the radius vector from the origin to any point on the surface of the scatterer; and $\mathbf{i}_{i}=\mathbf{a}-(\mathbf{a} \cdot \mathbf{n}) \mathbf{n}$.

If the receiver is at a very great distance from the body, if the body is finite, and if the incident magnetic field is of unit magnitude, then Eq. (2-1) can be expressed in the form

where

$$
\mathbf{H}_{s c}=\frac{e^{-i k R^{\prime}}}{R^{\prime}} \mathbf{F}(\beta)
$$

$$
\begin{aligned}
& \mathbf{F}(\beta)=\frac{i k}{2 \pi}\left[\left(\mathbf{n}_{0} \cdot \mathbf{a}\right) \mathbf{f}-\left(\mathbf{n}_{0} \cdot \mathbf{f}\right) \mathbf{a}\right], \\
& \mathbf{f}=\int_{\substack{\text { illuminated } \\
\text { region of body }}} \mathbf{n} \exp \left[-i k \mathbf{r} \cdot\left(\mathbf{n}_{0}+\mathbf{k}\right)\right] d s \\
&=\mathbf{i}_{x} I_{x}+\mathbf{i}_{y} I_{y}+\mathbf{i}_{z} I_{z},
\end{aligned}
$$

and $R^{\prime}=$ the distance from the origin to the receiver.

The radar cross section is given by ${ }^{11}$

$$
\sigma(\beta)=4 \pi\left[\left|F_{x}\right|^{2}+\left|F_{y}\right|^{2}+\left|F_{z}\right|^{2}\right] .
$$

The amount of energy received is proportional to the square of the magnitude of the scalar product of the vectors $\mathbf{H}_{s c}$ and $\mathbf{d}$, where $\mathbf{d}$ is the direction of the receiver polarization; thus it is convenient to define

${ }^{10} \mathrm{~J}$. A. Stratton, Electromangetic Theory (McGraw-Hill Book Company, Inc., New York, 1941), p. 466.

$\dagger$ The shadow curve is the locus of points on the body for which $\mathbf{k} \cdot \mathbf{n}=0$. This curve separates the portion of the body "seen" by the transmitter (i.e., the illuminated side) from the shadow side.

${ }^{11}$ K. M. Siegel and H. A. Alperin, Report No. UMM-87, Willow Run Research Center, University of Michigan (January, 1952).

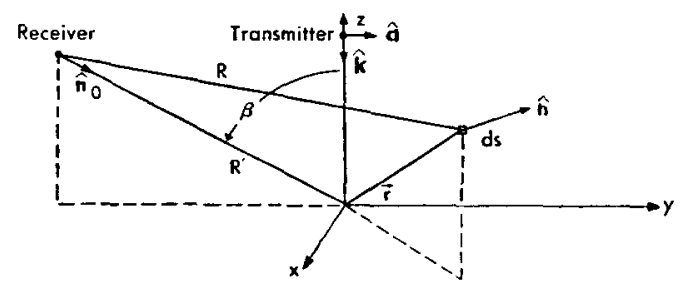

FIG. 2. Geometry for surfaces of revolution.

the effective cross section as

$$
\begin{aligned}
& \sigma_{e}(\beta)=4 \pi|\mathbf{F} \cdot \mathbf{d}|^{2} \\
& \sigma_{e}(\beta)=\frac{4 \pi}{\lambda^{2}}\left|\left(\mathbf{n}_{0} \cdot \mathbf{a}\right)(\mathbf{f} \cdot \mathbf{d})-\left(\mathbf{n}_{0} \cdot \mathbf{f}\right)(\mathbf{a} \cdot \mathbf{d})\right|^{2}
\end{aligned}
$$

If $\mathbf{d}$ is given by a vector whose components are proportional to the complex conjugates of the corresponding components of $\mathbf{F}$, then $\sigma_{e}(\beta)$ reduces to $\sigma(\beta)$.

If the body is a surface of revolution (the axis of symmetry taken to be the z-axis), if the receiver is restricted to be in the $y z$-plane with $y \leqslant 0$, and if, in addition, the transmitter is located on the $z$-axis $(z>0)$, then the geometry shown in Fig. 2 applies. With the situation as pictured in Fig. 2,

$$
\mathbf{n}_{0}=\mathbf{i}_{y} \sin \beta-\mathbf{i}_{z} \cos \beta, \text { and } \mathbf{k}=-\mathbf{i}_{z} .
$$

If the polarization of the incident wave is $\mathbf{a}=\mathbf{i}_{y}$ and if the surface is symmetric with respect to the $y z$-plane, then from Eq. (2-3)

where

$$
\sigma_{\mathfrak{a}=\mathbf{i} y}(\beta)=\frac{4 \pi}{\lambda^{2}}\left|I_{z}\right|^{2}
$$

$$
I_{z}=\int_{\substack{\text { illuminated } \\ \text { region of body }}} n_{z} \exp \left[-i k \mathbf{r} \cdot\left(\mathbf{n}_{0}+\mathbf{k}\right)\right] d s .
$$

In many cases, the integral $I_{z}$ can be evaluated easily with the aid of the following Double Stationary-Phase theorem (hereafter, this theorem will be referred to as the D.S.P. theorem) :

Theorem:

Let

If

$$
I=\int_{c}^{d} \int_{a}^{b} f(x, y) e^{i k g(x, y)} d x d y .
$$

(1) $f(x, y)=X(x) Y(y)$ is analytic in the region

$$
R=\{x, y \mid a \leqslant x \leqslant b, \quad c \leqslant y \leqslant d\},
$$

(2) $g(x, y)$ is analytic in $R$,

(3) there exists one, and only one, point $\left(x_{0}, y_{0}\right)$ in the interior of $R$, such that

$$
p_{0}=q_{0}=0 \text { and } r_{0} t_{0}-s_{0}^{2} \not \neq 0,
$$

where

$$
p=g_{x}, \quad q=g_{y}, \quad r=g_{x x}, \quad s=g_{x y}
$$


and

and

$$
t=g_{y y}
$$

(4) $X(\xi) / g_{\xi}(\xi, \eta)$ and $Y(\eta) / g_{\eta}(\xi, \eta)$ are of bounded variation for $(\xi, \eta)$ in $R$ but not in

$$
R^{\prime}=\left(x, y \mid x_{0}-\delta \leqslant x \leqslant x_{0}+\delta, y_{0}-\epsilon \leqslant y \leqslant y_{0}+\epsilon\right),
$$

then

$$
I=\frac{ \pm 2 i \pi e^{i k g\left(x_{0}, y_{0}\right)} f\left(x_{0}, y_{0}\right)}{k\left[r_{0} t_{0}-s_{0}^{2}\right]^{\frac{1}{3}}}+O\left(\frac{1}{k^{\frac{3}{2}}}\right) \text { as } k \rightarrow \infty .
$$

For a surface whose first and second derivatives are continuous, the essential contribution to the cross section for short wavelengths can be obtained from the integral formulation of the current-distribution method if the integration is performed by the method of stationary phase. If the integration is performed exactly, a result dependent upon wavelength is usually obtained. Since the application of the method of stationary phase and the physical-optics integrals depend on the parameter $(k l)^{-1}$, where $k=2 \pi / \lambda, l$ is the characteristic dimension of the body and $\lambda$ is the wavelength, it can be shown that the physical-optics result is a perturbation of the geometric-optics (or stationaryphase) result.

For a pointed body, the method of stationary phase cannot be applied directly for most values of $\beta$. The cross section predicted by geometric-optics is equal to $\pi R_{1} R_{2}$, where $R_{1}$ and $R_{2}$ are the two radii of curvature at the stationary-phase point. A stationary-phase point is one at which a plane of constant phase $\left(\mathbf{n}_{0}+\mathbf{k}\right) \cdot \mathbf{r}$ $=$ const, is tangent to the surface of the body. Hence, if a finite body has a point singularity, its cross section by geometric-optics would be zero. In this case, the physical-optics answer cannot be obtained from the geometric-optics answer by perturbation because there is no nonzero solution to perturb. A solution can, however, be obtained by integrating the surface integrals obtained exactly in one variable and by stationary phase in the other.

It is sometimes possible, by known methods, to solve the bistatic problem if the transmitter is located on the axis of symmetry but not otherwise. Since it is often of more interest to have the receiver located in this position, the reciprocity theorem of electromagnetic theory ${ }^{12}$ should be examined in the light of the approximation technic to be employed. This theorem allows the cross section to be determined for given positions of the transmitter and receiver if the problem has been solved with these positions interchanged.

With $\sigma_{e}(\beta)$ defined as in Eq. (2-4), it follows that when the wavelength becomes extremely short, all the contributions to $f$ cancel out except those from the immediate vicinity of the stationary-phase point on the side of the body toward the transmitter. Clearly

12 S. A. Schelkunoff, Electromagnetic Waves (D. Van Nostrand Company, Inc., New York, 1943). $\mathbf{n}=\left(\mathbf{n}_{0}+\mathbf{k}\right) /\left|\mathbf{n}_{0}+\mathbf{k}\right|$ at a stationary-phase point, and, for extremely short wavelengths, $\mathrm{f}=A\left(\mathbf{n}_{0}+\mathbf{k}\right)$ where $A$ is not changed by an interchange of transmitter and receiver. Therefore, in this limiting case, Eq. (2-4) becomes

$\sigma_{e}(\beta) \approx \frac{4 \pi}{\lambda^{2}}|A|^{2}\left|\left(1+\mathbf{n}_{0} \cdot \mathbf{k}\right)(\mathbf{a} \cdot \mathbf{d})-\left(\mathbf{n}_{\mathbf{0}} \cdot \mathbf{a}\right)(\mathbf{k} \cdot \mathbf{d})\right|^{2}$.

If $\mathbf{n}_{0}$ and $\mathbf{k}$ are interchanged, and simultaneously $\mathbf{a}$ and $\mathbf{d}$ are interchanged Eq. (2-6) is unaffected. Hence, reciprocity is obtained for the current distribution method in the limit of extremely short wavelengths.

When a wavelength-dependent expression for the cross section is obtained by the current-distribution method, complete reciprocity is not obtained. However, since the exact theory shows that there is reciprocity, whenever the current-distribution method yields an appropriate wavelength-dependent formula for one case, the result can be considered to be an adequate approximation for the reciprocal case.

\section{CROSS-SECTION FORMULAS FOR VARIOUS SURFACES}

In this section the method discussed in the preceding section is applied to several geometrical configurations. The method is applied directly in the cases of the prolate spheroid and the sphere in Section 3.1. A discussion of the application of the method to a finite cone appears in Section 3.2, and to an ogive in Section 3.3. Section 3.4 contains the results obtained for an infinite paraboloid which can be shown ${ }^{13}$ to be the exact solution to the problem. Although the semi-infinite cone is a surface which does not satisfy the basic assumptions referred to in Section 2, the results obtained through the formal application of the method (with an Abelian limit procedure added) are given in Section 3.5. The results obtained through a formal application of the LunebergKline method are also given. Section 3.6 contains an extension of the method of Section 2, as does Section 3.7. The elliptic cylinder is discussed in Section 3.6 and a special case (in which the integration can be done exactly) for the spheroid appears in Section 3.7.

\subsection{The Prolate Spheroid and the Sphere}

The direct application of the method described in Section 2 to the problem of determining $\sigma \mathrm{a}=\mathrm{i}_{y}(\beta)$ for the prolate spheroid given by

$$
\frac{x^{2}+y^{2}}{B^{2}}+\frac{z^{2}}{A^{2}}=1
$$

and for the sphere

$$
x^{2}+y^{2}+z^{2}=R_{0}^{2}
$$

yields the following geometric-optics results:

${ }^{13}$ C. E. Schensted, J. Appl. Phys. 26, 306 (1955). 
For the prolate spheroid,

$$
\begin{gathered}
\sigma_{\mathbf{a}=\mathrm{i}_{\nu}}(\beta)=\left[4 \pi B^{4} / A^{2}\right]\left[(1+\cos \beta)+\frac{B^{2}}{A^{2}}(1-\cos \beta)\right]^{-2}, \\
\beta<\pi .
\end{gathered}
$$

For the sphere,

$$
\sigma \mathrm{a}=\mathbf{i}_{y}(\beta)=\pi R_{0}^{2}, \quad \beta<\pi .
$$

These geometric-optics results were obtained by using the D.S.P. theorem.

$\sigma_{\mathrm{a}}=i_{y}(\beta)$ for both the prolate spheroid and the sphere were also determined by integrating once exactly and once by analog machine methods. The analog computing equipment consisted of standard Reeves analog computor units. The "analog evaluation" was performed for the special cases given by $A=10 B$ and $k B=100$ for the prolate spheroid and $k R_{0}=100$ for the sphere. Good agreement between the analog results and the D.S.P. results was found to exist in the range $0^{\circ} \leqslant \beta$ $<160^{\circ}$ for the prolate spheroid and in the range $0^{\circ} \leqslant \beta<120^{\circ}$ for the sphere. A graphical representation of these results appears in Fig. 3.

\subsection{The Finite Cone}

For a finite cone of slant length $r_{0}$ and one-half cone angle $\gamma$, the application of Eq. (2-5) yields the formula

$$
\sigma_{\mathrm{a}=\mathrm{i}_{y}}(\beta) \approx \frac{4 \pi r_{0}^{2} \sin 4 \gamma}{\left(b^{2}-c^{2}\right)^{2}}\left\{c^{2} J_{0}^{2}\left(k r_{0} b\right)+b^{2} J_{1}^{2}\left(k r_{0} b\right)\right\}
$$

where

$$
b=\sin \beta \sin \gamma, \quad c=\cos \gamma(1+\cos \beta),
$$

and $\beta$ is restricted by the inequality $0 \leqslant \beta<\pi-2 \gamma$. This formula was obtained by integrating the integral $I_{z}$

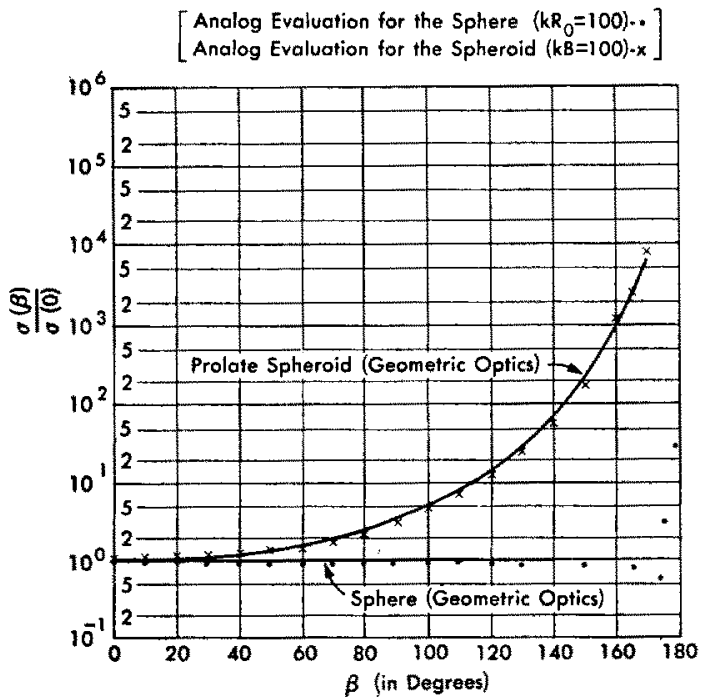

FIG. 3. Cross sections of a sphere of radius $R_{0}$ and of a prolate spheroid for which $A=10 B$ as a function of the angle $\beta$.

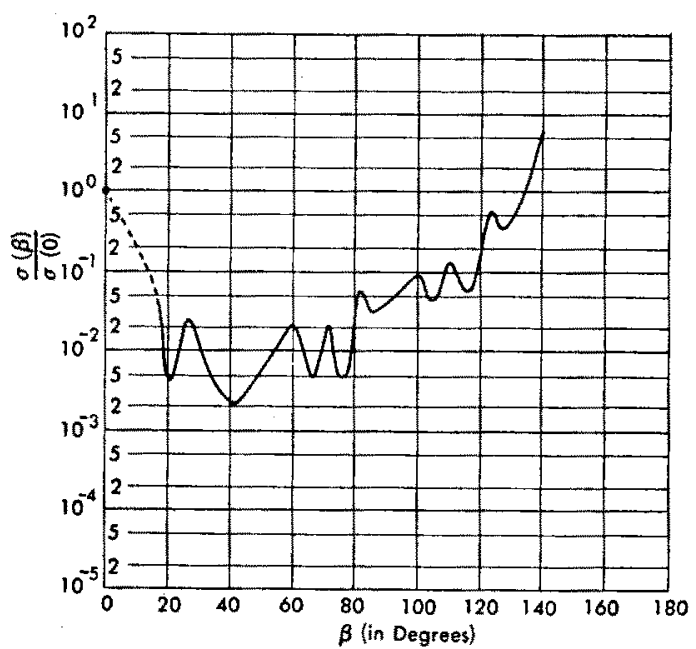

FIG. 4. Cross section of a finite cone of half-angle $15^{\circ}$ and slant length equal to $100 \mathrm{~N} / \pi$.

once by parts and once by stationary phase and then retaining only the dominant term (for large $k$ ).

A graphical representation of Eq. (3-3) appears in Fig. 4 for the special case of $\gamma=15^{\circ}$ and $\pi r_{0} / \lambda=100$.

It should be pointed out that the sharp edge of the finite cone contradicts the assumptions of the currentdistribution method. Thus, the result obtained here for the finite cone may not be as good an approximation to the exact cross section as the results obtained for other bodies.

\subsection{The Ogive}

The term "ogive" is not defined uniformly in the literature. For example, Hobson ${ }^{14}$ refers to the figure obtained by revolving a minor arc of a circle about its chord as a spindle, whereas Hansen and Schiff ${ }^{15}$ use the term spindle to describe the arc of a parabola revolved about its chord. Others use the term ogive as a general term of which both of the above configurations are examples. In the work presented here the term ogive refers to the minor arc of a circle revolved about its chord (Hobson's spindle) and the term spindle will be used for the parabolic arc revolved about its chord (Hansen and Schiff).

An ogive of length $L$, maximum diameter $d$, and halfangle $\alpha$ (using the cylindrical coordinates $w, \phi, z$ ) is given by the equation

$$
(w+h)^{2}+z^{2}-P^{2}=0
$$

with

$$
\begin{aligned}
& P=\text { radius of the generating circular arc, } \\
& |z| \leqslant\left(P^{2}-h^{2}\right)^{\frac{1}{2}}=L / 2, \\
& h \leqslant w+h \leqslant P,
\end{aligned}
$$

${ }^{14} \mathrm{E}$. W. Hobson, The Theory of Spherical and Ellipsoidal Harmonics (The Cambridge University Press, New York, 1931)

${ }^{15}$ W. W. Hansen and L. I. Schiff, "Theoretical study of electromagnetic waves scattered from shaped metal surfaces," Quarterly Report No. 3 (Microwave Laboratory, Department of Physics, Stanford University, May, 1948). 
and

$$
\alpha=\arccos (h / P) \text {. }
$$

Direct use of Eq. (2-5) (integrating once exactly and once by parts) implies that the contributions from the tip of the ogive and from the shadow rim are of the same order of magnitude. However, Hansen and Schiff, who obtained a similar result in their work on the spindle (see reference 15), showed by a more careful consideration of the contribution of the shadow rim that, in fact, the contribution of the shadow rim (or rather, the penumbra) is of a smaller order of magnitude than that of the point and that the "correct" answer can be obtained by merely evaluating the integral at the "tip." By applying an analysis similar to Hansen and Schiff's penumbra analysis to the ogive problem, i.e., ignoring the contribution from the shadow rim, it is found that $\sigma(0)$ for the ogive is given by

$$
\sigma(0)=\frac{\lambda^{2} \tan ^{4} \alpha}{16 \pi}, \quad b_{0}-\frac{1}{1}<\alpha<\frac{\pi}{2}-b_{0}^{-\frac{1}{2}}
$$

where $b=b(\beta)=k p(1+\cos \beta)$, and $b_{0}=b(0)$. It should be pointed out that this result agrees with the electromagnetic theory answer for a semi-infinite cone having either a large cone-angle or a small cone-angle (see references 3 and 4 ).

Assuming that the tip will also dominate for most of the values of $\beta$ of interest (i.e., for $0 \leqslant \beta<\pi-2 \alpha-\left|\beta_{0}(\lambda)\right|$ where $\beta_{0}(\lambda) \rightarrow 0$ as $\lambda \rightarrow 0$ ) as it did for $\beta=0$ and using an approach similar to that employed in obtaining (3-4) the tip formula for the ogive becomes

$$
\sigma_{\mathfrak{a}=\mathbf{i}_{y}}(\beta)=\frac{\lambda^{2} \tan ^{4} \alpha\left[1-\tan ^{2} \alpha \tan ^{2}(\beta / 2)\right]^{-3}}{16 \pi \cos ^{3}(\beta / 2)},
$$

with $0 \leqslant \beta<\pi-2 \alpha$. The application of the D.S.P. theorem yields

$$
\sigma_{\mathbf{a}=\mathbf{i}_{\nu}}(\beta)=\frac{\pi L^{2}[\sin (\beta / 2)-\cos \alpha]}{4 \sin ^{2} \alpha \sin (\beta / 2)}, \quad \pi-2 \alpha<\beta<\pi .
$$

$\sigma(\beta)$ was evaluated on analog equipment for the case defined by $\alpha=15^{\circ}$ and $\pi d / \lambda=100$. The nature of the integrand made it difficult to obtain reliable results over the entire range in $\beta$; however, fairly reliable results were obtained in the interval $\pi / 2<\beta \leqslant \pi$.

In summary, $\sigma a=i_{y}(\beta)$ for the ogive has been determined by

(1) the "tip" method of determination

$$
\left(0 \leqslant \beta<\pi-2 \alpha-\left|\beta_{0}(\lambda)\right|\right),
$$

(2) the analog evaluation (reliable for $\beta>\pi / 2$ ), and

(3) the D.S.P. method (valid for $\pi-2 \alpha<\beta<\pi$ ).

Examination of these results indicates that if a single curve were to be fitted through these data, it would follow the tip formula almost up to the $\pi-2 \alpha$ value

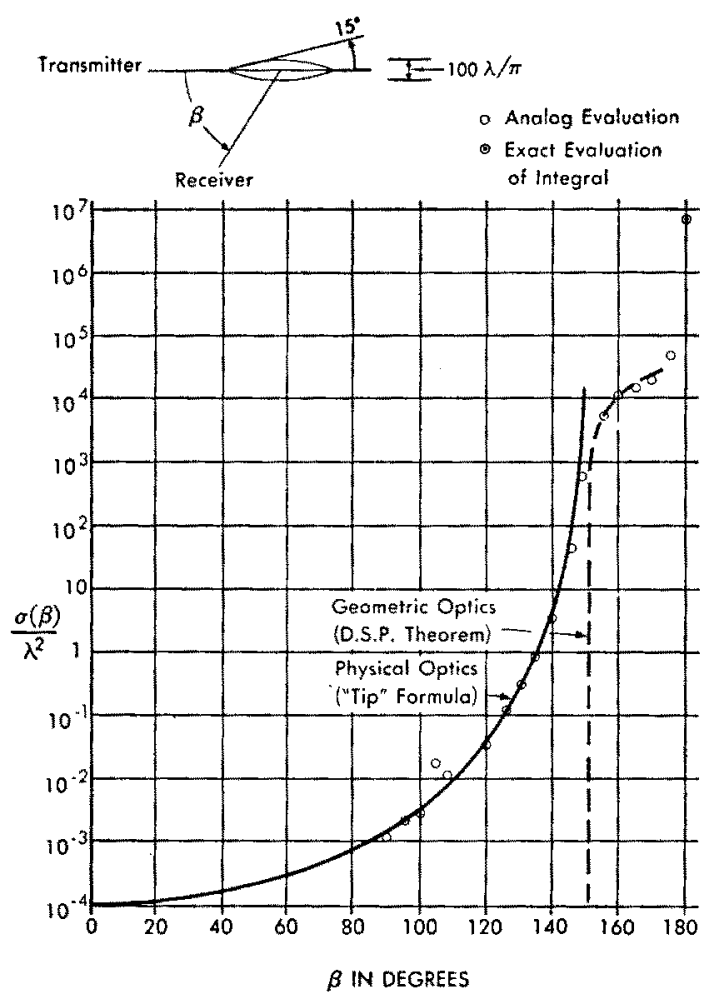

Frg. 5. Cross section of an ogive of half-angle $15^{\circ}$ and maximum diameter equal to $100 \mathrm{~N} / \pi$.

of $\beta$ and then follow the trend indicated by the analog evaluations and the D.S.P. result. The analog evaluations and the D.S.P. result are in close agreement in the range $155^{\circ} \leqslant \beta \leqslant 175^{\circ}$ for the case considered on analog. A graphical representation of these results appears in Fig. 5.

\subsection{The Paraboloid}

Although the method given in Section 2 is based in part upon the assumption that the surface involved is finite, the application of Eq. (2-5) to the paraboloid defined by

$$
x^{2}+y^{2}=-4 P z
$$

yields an infinite integral which can be evaluated exactly. The method yields the formula,

$$
\sigma_{\mathrm{a}}=\mathrm{i}_{y}(\beta)=16 \pi P^{2} /(1+\cos \beta)^{2}=4 \pi P^{2} \sec ^{4}(\beta / 2) .
$$

Because of the relative simplicity of this formula, no graphical representation is given. It is shown (see reference 13) that (3-7) gives the exact cross section for axially symmetric scattering from a paraboloid of revolution.

\subsection{The Semi-Infinite Cone}

If the method of Section 2 is applied formally (the assumptions upon which Eq. (2-3) are based involve assuming that the body is finite in size and does not contain a point), and the integral involved is evaluated 

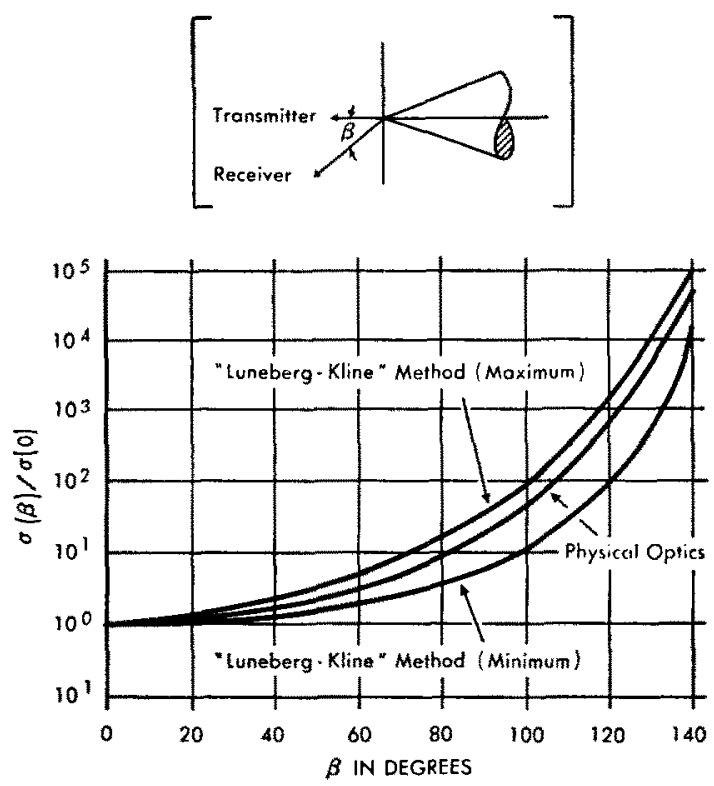

FIG. 6. Cross section of a semi-infinite cone of half-angle $15^{\circ}$.

with the aid of an Abelian limit process, the following formula for a semi-infinite cone is obtained:

$$
\sigma(\beta)=\frac{\lambda^{2} \tan ^{4} \gamma}{16 \pi} \cdot \frac{2(1+\cos 2 \gamma)^{3}}{(1+\cos \beta)(\cos \beta+\cos 2 \gamma)^{3}}
$$

where $\gamma=\frac{1}{2}$ cone-angle and $0 \leqslant \beta<\pi-2 \gamma$.

The Luneberg-Kline method cannot legitimately be applied to the cone problem, since the radius of curvature at the nose of the cone is zero. However, if the method is applied formally, the expression obtained has Eq. (3-8) as its first term. The "Luneberg-Kline answer" agrees with the intuitive expectation that the cross section should be a maximum in the plane of electric polarization. Also, the cross section obtained by the formal application of the Luneberg-Kline method increases as a function of the separation between transmitter and receiver (transmitter on the cone axis) in the expected fashion.

The formula obtained by this method is

$$
\begin{array}{r}
\sigma(\beta)=\frac{\lambda^{2} \tan ^{4} \gamma}{16 \pi} \frac{2(1+\cos 2 \gamma)}{(1+\cos \beta)(\cos 2 \gamma+\cos \beta)} \\
\quad \times\left[\left(\frac{1+\cos 2 \gamma}{\cos 2 \gamma+\cos \beta}\right)^{2}+2\left(\frac{1+\cos 2 \gamma}{\cos 2 \gamma+\cos \beta}\right)\right. \\
\left.\quad \times\left(\frac{1-\cos \beta}{1+\cos \beta}\right) \cos 2 \phi+\left(\frac{1-\cos \beta}{1+\cos \beta}\right)^{2}\right]
\end{array}
$$

where $\phi$ is the angle between the plane of polarization of the incident field, and the plane containing the axis of the cone and the receiver,
For backscattering, $\beta=0$, this reduces to

$$
\sigma(0)=\lambda^{2} \tan ^{4} \gamma / 16 \pi
$$

which is just the well-known physical-optics result for backscattering. It has previously been shown in reference 3 , that the physical-optics answer agrees with the exact backscattering answer both for large cone angles and for small cone angles.

From Eq. (3-9) it can be seen that the maximum cross section is obtained when $\phi=0$ and $\phi=\pi$, while the minimum cross section is obtained for $\phi=\pi / 2$ and $\phi=3 \pi / 2$. The physical-optics bistatic cross section of a semi-infinite cone, given above in Eq. (3-8), is independent of $\phi$. Since (3-9) is not independent of $\phi$ it is clear that the two solutions do not agree exactly. Examination of (3-8) and (3-9) f† however, shows that the physical-optics answer agrees very well with the answer obtained by the use of the Luneberg-Kline method and that it lies approximately halfway between the maximum and minimum "Luneberg-Kline" curves. This is shown in Fig. 6.

\subsection{The Elliptic Cylinder}

Consider an elliptic cylinder with semimajor axis $a$ and semiminor axis $b$ oriented with respect to the transmitter and the receiver as shown in Fig. 7 . The angular positions of the transmitter and receiver are designated by $\theta_{t}, \phi_{t}$ and $\theta_{r}, \phi_{r}$ respectively.

By definition, the radar cross section is given by

$$
\sigma=4 \pi\left[\left|F_{x}\right|^{2}+\left|F_{y}\right|^{2}+\left|F_{z}\right|^{2}\right]
$$

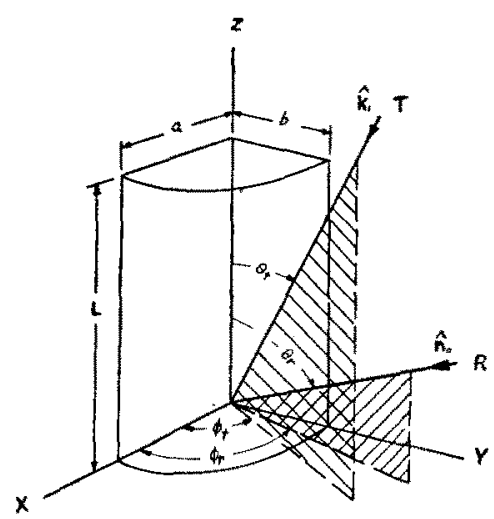

FIG. 7 . Geometry for the elliptic cylinder.

$\dagger \dagger$ Note added in proof.-Felsen (Report No. R-362-54, PIB-296, Microwave Research Institute of Brooklyn) has obtained the exact answer for small angle cones. His result agrees with Eq. (3-8) but not with $\mathrm{Eq}$. (3-9) when the transmitter is on the cone axis. This is in agreement with a conjecture made by Siegel (see reference 2) that physical optics give the correct vector cross section for infinite bodies with an axis of symmetry in the direction of Poynting's vector. 

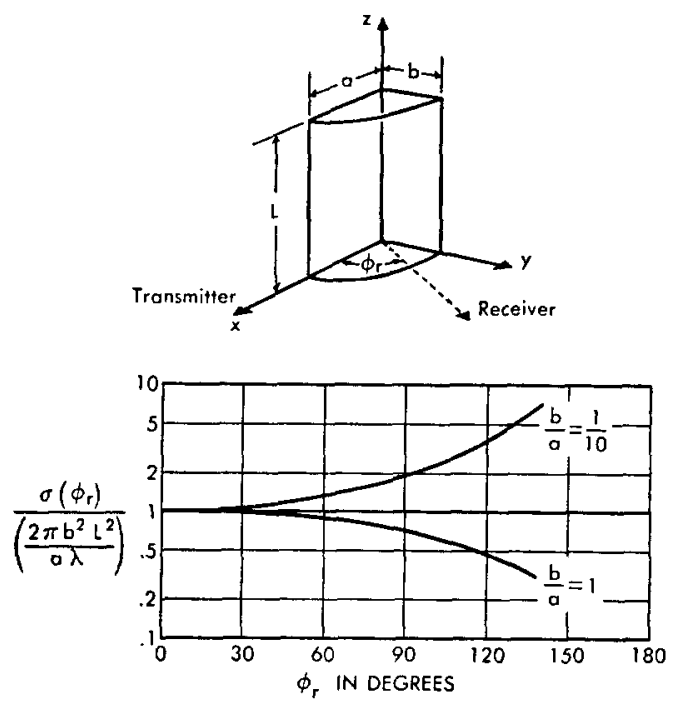

FIG. 8. Bistatic cross section of an elliptic cylinder for the case of transmitter and receiver in a plane perpendicular to the axis of the cylinder.

where

$$
\begin{gathered}
\mathbf{F}\left(\theta_{t}, \theta_{r}, \phi_{t}, \phi_{r}\right) \\
=\frac{-i k}{2 \pi}\left(\mathbf{n}_{0} \cdot \mathbf{a}\right) \int_{\begin{array}{c}
\text { illuminated } \\
\text { portion of } \\
\text { the surface }
\end{array}} \exp \left[i k \mathbf{r} \cdot\left(\mathbf{k}+\mathbf{n}_{0}\right)\right] \mathbf{n} d s \\
+\frac{i k \mathbf{a}}{2 \pi} \int_{\begin{array}{c}
\text { illuminated } \\
\text { portion of } \\
\text { the surface }
\end{array}} \exp \left[i k \mathbf{r} \cdot\left(\mathbf{k}+\mathbf{n}_{0}\right)\right]\left(\mathbf{n}_{0} \cdot \mathbf{n}\right) d s,
\end{gathered}
$$

and all the other parameters are as defined in Section 2. The problem of finding the cross section is the problem of evaluating the integrals. This evaluation can be accomplished most simply in elliptic cylindrical coordinates $(\xi, \eta, z)$.

Integrating with respect to $z$ first and then by the method of stationary phase, we obtain

$\sigma\left(\theta_{r}, \theta_{t}, \phi_{r}, \phi_{t}\right)=\frac{a^{2} b^{2} \lambda\left|e^{i k D L}-1\right|^{2}}{\pi D^{2}\left[(A a)^{2}+(B b)^{2}\right]^{\frac{3}{2}}}\left\{G_{1}{ }^{2}+G_{2}{ }^{2}+G_{3}{ }^{2}\right\}$,

where

$G_{1}=A\left(a_{y} \sin \theta_{r} \sin \phi_{r}+a_{z} \cos \theta_{r}\right)-B\left(a_{x} \sin \theta_{r} \sin \phi_{r}\right)$,

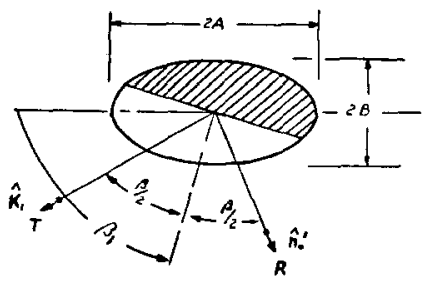

FIG. 9. Geometry for the prolate spheroid.
$G_{2}=a_{z} \sin \theta_{r}\left(A \cos \phi_{r}+B \sin \phi_{r}\right)$,

$G_{3}=B\left(a_{x} \sin \theta_{r} \cos \phi_{r}+a_{z} \cos \theta_{r}\right)-A\left(a_{y} \sin \theta_{r} \cos \phi_{r}\right)$,

$A=\sin \theta_{t} \cos \phi_{i}+\sin \theta_{r} \cos \phi_{r}$,

$B=\sin \theta_{t} \sin \phi_{t}+\sin \theta_{r} \sin \phi_{r}$,

and

$D=\cos \theta_{t}+\cos \theta_{r}$.

Figure 8 is a plot of the above relationship for the special case defined by $\theta_{r}=\theta_{t}=\pi / 2$ and $\phi_{t}=0$. For the special case of backscattering in which $\phi_{t}=\phi_{r}=\phi$ and $\theta_{t}=\theta_{\tau}=\theta(3-11)$ becomes

$$
\sigma(\theta, \phi)=\frac{a^{2} b^{2} \lambda\left|e^{i k D L}-1\right|^{2} \sin ^{4} \theta}{\cos ^{2} \theta\left[(A a)^{2}+(B b)^{2}\right]^{\frac{2}{2}}} .
$$

\subsection{The Prolate and Oblate Spheroids for Particular Choices of Transmitter and Receiver Directions}

In Section 3.1 the bistatic cross section of a prolate spheroid was determined for the case in which the transmitter is located on the axis of symmetry. In this

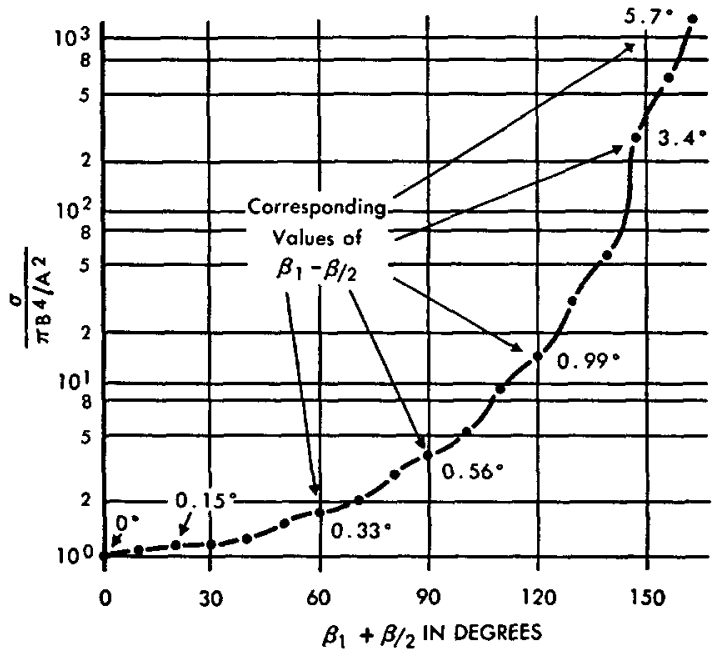

FIG. 10. Bistatic cross section of a prolate spheroid $(k A=25$ and $k B=2.5)$.

section it will be shown that for each transmitter position (not necessarily on the symmetry axis) there is one particular receiver direction (or two when use is made of reciprocity) for which the current-distribution integral can be evaluated exactly, both for a prolate and an oblate spheroid.

The geometry used in this evaluation is shown in Fig. 9. The axis of the spheroid, $\mathbf{n}_{0}{ }^{\prime}$ and $\mathbf{k}_{1}$ all lie in the same plane and the angle between the axis and the normal to the phase planes is $\beta_{1}$. The angular separation between the transmitter, $T$, and the receiver, $R$, is $\beta$.

If $S$ is the surface, $S^{*}$ is a second surface bounded by the shadow curve, and $V$ is the volume contained 
between $S$ and $S^{*}$, then the integral over the surface $S$, which one obtains in applying the current-distribution method, may be expressed as a sum of an integral over $V$ and an integral over $S^{*}$. For a spheroid, $S^{*}$ can always be chosen as the interior of an ellipse so that the second integral is easy to evaluate. If $S^{*}$ lies in a phase plane, the first integral is also easy to evaluate. This case is the particular case for which the crosssection integrals can be evaluated exactly.

Carrying out this method of approach yields

$$
\sigma=\frac{\pi A^{2} B^{4}}{\rho_{0}^{4}}\left[1-2 \frac{\sin (2 M)}{2 M}+\frac{\sin ^{2}(M)}{M^{2}}\right]
$$

where

$$
M=k \rho_{0} \cos (\beta / 2), \text { and } \rho_{0}^{2}=A^{2} \cos ^{2} \beta_{1}+B^{2} \sin ^{2} \beta_{1} .
$$

Equation (3-13) applies only when the shadow curve lies in a phase plane. This condition is satisfied when

$$
\tan (\beta / 2)=\frac{A^{2}-B^{2}}{\rho_{0}^{2}} \sin \beta_{1} \cos \beta_{1} .
$$

\begin{tabular}{|c|c|c|c|}
\hline Surface & $\begin{array}{l}\text { Orientation of } \\
\text { surface and radat }\end{array}$ & Cross section & $\begin{array}{l}\text { Code to } \\
\text { symbols }\end{array}$ \\
\hline Torus & $\begin{array}{l}\text { Direction of propa- } \\
\text { gation is parallel } \\
\text { to the axis of the } \\
\text { totus }\end{array}$ & $a=8 \pi^{3} R_{k}^{2} R_{\alpha} X^{-1}$ & $\begin{aligned} & R_{1}= \text { distance to } \\
& \text { center of } \\
& \text { ring. } \\
& R_{2}= \text { tadius of } \\
& \text { ring. }\end{aligned}$ \\
\hline $\begin{array}{l}\text { Flat } \\
\text { piate }\end{array}$ & $\begin{array}{l}\text { Direction of propa- } \\
\text { gation is normal } \\
\text { to surface }\end{array}$ & $\sigma=4 x W^{a} a^{2} \lambda^{-2}$ & $\begin{array}{c}W=\text { width of } \\
\text { plate. } \\
H=\text { height of } \\
\text { plate. }\end{array}$ \\
\hline $\begin{array}{l}\text { Large } \\
\text { disk }\end{array}$ & $\begin{array}{l}\text { Angle between di- } \\
\text { rection of propa- } \\
\text { gation and normal } \\
\text { to disk given by } \theta\end{array}$ & $\theta=\frac{4 \pi}{\lambda^{2}}\left[\frac{2 \pi a^{2} \cos \theta f_{1}(x)}{x}\right]^{2}$ & $\begin{array}{l}a=\text { radius of } \\
\text { disk. } \\
x=\frac{4 \pi c}{\lambda} \sin \theta\end{array}$ \\
\hline
\end{tabular}

TABLE I. Other approximate monostatic cross-section formulas.

The maximum separation between transmitter and receiver which is obtainable is given by

$$
\tan (\beta / 2)=\frac{A^{2}-B^{2}}{2 A B} \text { and } \tan \left(\beta_{1}\right)=A / B
$$

These results are illustrated in Fig. 10 for the special case given by $k B=2.5$ and $k A=25$.

The method used in this section could also be applied to other quadric surfaces. For example, special cases could be integrated exactly for an ellipsoid having three nonequal axes.

\section{8. $\sigma(3)$ for Other Surfaces}

The methods discussed on the preceding pages may

\begin{tabular}{|c|c|c|}
\hline Surface & Equation of surface & $\sigma_{\mathrm{a}}=\mathrm{f}_{y}(\beta)$ \\
\hline Ellipsoid & $\frac{x^{2}}{A^{2}}+\frac{y^{2}}{B^{2}}+\frac{z^{2}}{C^{2}}=1$ & $\begin{array}{c}\frac{4 \pi A^{3} B^{2}}{C^{2}}[(1+\cos \beta) \\
\left.+\frac{B^{2}}{C^{2}}(1-\cos \beta)\right]^{-2} \\
\beta<\pi\end{array}$ \\
\hline $\begin{array}{l}\text { One branch of } \\
\text { hyperboloid } \\
\text { of two sheets }\end{array}$ & $z=-C\left[1+\frac{x^{2}}{A^{2}}+\frac{y^{2}}{B^{2}}\right]^{2}$ & $\begin{array}{c}\frac{4 \pi A^{2} B^{2}}{C^{2}}[(1+\cos \beta) \\
\left.-\frac{B^{2}}{C^{2}}(1-\cos \beta)\right]^{-2} \\
\beta<2 \arctan (B / C)\end{array}$ \\
\hline $\begin{array}{l}\text { Elliptic para- } \\
\text { boloid (axis } \\
\text { of symmetry } \\
\text { is z-axis) }\end{array}$ & $\frac{x^{2}}{A^{2}}+\frac{y^{2}}{B^{2}}=-\frac{z}{C}$ & $\frac{\pi A^{2} B^{2}}{C^{2}(1+\cos \beta)^{2}}$ \\
\hline $\begin{array}{l}\text { Elliptic para- } \\
\text { boloid (axis } \\
\text { of symmetry } \\
\text { is } y \text {-axis) }\end{array}$ & $\frac{x^{2}}{A^{2}}+\frac{z^{2}}{C^{2}}=+\frac{y}{B}$ & $\begin{array}{c}\frac{\pi A^{2} C^{2}}{B^{2}(1-\cos \beta)^{2}} \\
\beta>0\end{array}$ \\
\hline
\end{tabular}
be applied to a variety of other surfaces. Tables I and
TABLE $\Pi$ I. Other approximate bistatic cross-section formulas.

(Geometry is as shown in Fig. 2.)

II contain formulas which are readily obtainable upon the application of these techniques.

\section{CONCLUSION}

Bistatic radar cross sections have been obtained for simple shapes by optics approximations. It would be desirable to be able to compare these results with experiment. Unfortunately, very little experimental data exist. With these data absent one must examine the results in the light of previous experience. Considering the diffraction of electromagnetic waves through an aperture, it is well known that optics results for many applications yield poor approximations. On the other hand the nose-on radar cross section of a cone obtained by the optics approximation is in close agreement with, and possibly identical to, the exact solution for large and small cone angles (see references 3 and 4 ) and the cross section of the paraboloid for axially symmetric incident radiation is found exactly by an optics approximation. In the next paper evidence is given for the use of physical-optics approximations for general infinite bodies of revolution.

The results found in this paper should only be considered useful when the wavelength is small with respect to the major dimensions of the body. Questions as to the order of magnitude of the error must be deferred until more experimental results or further exact solutions have been obtained. 